See discussions, stats, and author profiles for this publication at: https://www.researchgate.net/publication/307552064

\title{
A Direct Numerical Simulation Estimation of Viscous Energy Dissipation Rate in Taylor Vortex Reactor
}

Conference Paper · January 2016

DOI: 10.2316/P.2016.840-032

CITATION

1

4 authors, including:

\section{Harminder Singh}

Universidade Federal de São Carlos

19 PUBLICATIONS 86 CITATIONS

SEE PROFILE

Roberto de Campos Giordano

Universidade Federal de São Carlos

197 PUBLICATIONS 2,055 CITATIONS

SEE PROFILE

Some of the authors of this publication are also working on these related projects:

Project TheTaCo View project

Project Techno-economic-environmental analysis of the use of vinasse biogas in different biorefinary configurations View project
READS

82

Claudio Suazo

Universidade Federal de São Carlos

2 PUBLICATIONS 5 CITATIONS

SEE PROFILE 


\title{
A DIRECT NUMERICAL SIMULATION ESTIMATION OF VISCOUS ENERGY DISSIPATION RATE IN TAYLOR VORTEX REACTOR
}

\author{
Harminder Singh ${ }^{\mathrm{a}, \mathrm{b}}$, Claudio Alberto Torres Suazo ${ }^{\mathrm{a}}$, Roberto de Campos Giordano ${ }^{\mathrm{a}}$, Alain Linéb \\ a Universidade Federal de São Carlos \\ Rodovia Washington Luís, km 235 - SP-31, São Carlos - São Paulo - Brasil. \\ b Institute National des Sciences Appliquées \\ LISBP,Université de Toulouse, CNRS, INRA, INSA, Toulouse-France.

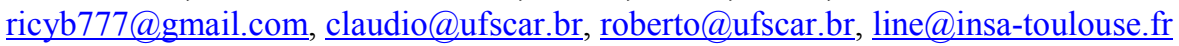

\begin{abstract}
This work presents a study of the direct numerical simulation (DNS) model in a Taylor-Vortex reactor (TVR) to estimate viscous energy dissipation rate (VEDR) after validating with an experimental 2D particle image velocimetry (PIV) study conducted with a $16 \mathrm{M}$ pixel camera. The VEDR was separated into its mean flow kinetic energy (MFKE) and turbulent kinetic energy components (TKE). The structure of the mean and fluctuating radial and tangential velocity components was mostly well predicted and the VEDR of mean flow kinetic energy was estimated well by the DNS model in comparison with the PIV estimations. The three secondorder radial gradients accounted for more than $70 \%$ of the VEDR through-out the reactor, clearly indicating the necessity of highly refined radial mesh.
\end{abstract}

\section{KEY WORDS}

3-Dimensional modelling, mathematical modelling, viscous energy dissipation rate.

\section{Introduction}

The cultivation of animal cells (including human) in in vitro in a bioreactor requires an environment as similar as possible to the natural cell environment. One of the most crucial factors that play a significant role in the design of a bioreactor using animal cells is that the animal cells do not possess a cell wall, unlike bacteria, fungi and plant cells; therefore, it is imperative to develop bioreactors that generate hydrodynamic forces milder than those found in bioreactors cultivating microbes [1]. In recent years, Taylor-Vortex reactor (TVR) has emerged as a choice for culturing cells [2-3]. These researchers successfully cultured various animal and insect cell lines, namely Chinese Hamster Ovaries (CHO), Human Embryonic Kidney, rat bone marrow stroma and Sf-9 Spodoptera frugiperda, in suspension in a TVR. The oxygen is easily provided through gas-permeable membranes in these reactor types thus reducing the hydrodynamic stress due to aeration by bubbling $[5,8]$. Another advantage of the TVR is the possibility of scale-up to an industrial level reactor, thus making it a serious contender for culturing animal cells.
On the other hand, numerous parameters have been used to characterize the effect of hydrodynamic forces on the cells, but none comes closer to the turbulence energy dissipation rate, which has been termed as the ideal parameter to characterize the potential of hydrodynamic stresses to damage cells by the team of Prof. Jeffrey Chalmers from the Ohio state university in USA through various meticulous studies [4-10]. They used viscous energy dissipation rate (VEDR) because it is intrinsic to any moving fluid and a scalar value, in addition to accounting for both shear and extensional components of three-dimensional flow. Furthermore, depending on the system geometry and the flow regime, the VEDR can be determined experimentally or obtained via computer simulations.

Till late nineties the major source of knowledge about the flow structures and hydrodynamics came through experimental methods in view of constrained computational resources. Mavros [11] cited the various experimental techniques that are practiced for flow visualization and analyzing hydrodynamics, and stated that particle image velocimetry (PIV) and laser-Doppler anemometry (LDA) have become the predominant methods due to their relative easy-to-use techniques. The PIV method has been implemented a number of times in the TVR, but mostly only velocity and vortex structures have been examined [12-14]. Van Hout \& Katz [15] and Tokgoz et al. [16] presented turbulence parameters, such as Reynolds stresses and VEDR, using the PIV method, but their spatial resolution was limited for turbulent Reynolds number. Evolution in these experimental methods has not been sufficient enough to capture the smallest scales at turbulent Reynolds number [17]. It could be that the shear practicality and flexibility of numerical methods have shifted the weight towards computational side to study the hydrodynamics. Nonetheless, the experimental methods are necessary in order to validate the numerical results.

Significant recent advances in the computational field has enabled the researchers to use the direct numerical simulation (DNS) model to examine the hydrodynamics at high Reynolds number in a simple geometry, such as TVR. In a TVR, consisting of two concentric cylinders, flow can be generated by rotating one or both cylinders in co- or counter-current directions. The field of study in 
such a reactor is the gap-width, the distance between the inner and outer cylinder, which is of the order of only few centimeters for laboratory scale reactors. As a result, obtaining a mesh which is nearer to the smallest Kolmogorov scale in order to capture around $90 \%$ of the eddies is within reach. Indeed, there has been a surge in the studies of the TVR using the DNS model in recent years [18-22].

Bilson \& Bremhorst [18] showed a detailed analysis of velocity fluctuations, turbulence kinetic energy (TKE), Reynolds stress budget and one-dimensional energy spectra for a radius ratio, $\eta_{\mathrm{rr}}$, of 0.617 and an aspect ratio, $\Gamma$, of 4.58. The main focus of the DNS study of Dong [19] was to demonstrate the effect of the variation in Reynolds number on the Gortler vortices and herringbone-like streaks that develop near the cylinder walls, and partly the statistical features of turbulence in TVR. Pirro \& Quadrio [20] explained the numerical method that they developed extending the work of Quadrio \& Lichini [23] to use the DNS model in fully turbulent regime at Reynolds number of 10500. After validating with numerical and experimental results, they discussed mean velocity and root mean square value of velocity fluctuations profiles. Their major observation was the identification of two distinct sources of fluctuations, namely: 1) curvature related large-scale vortices, and 2) small scale fluctuations produced by the wall turbulence cycle. The objective of using the DNS model by Douaire [21] was to be able to explain the cell behavior with respect to the TVR hydrodynamics at different Reynolds number. They used the DNS model to estimate various volumetric distributions, such as that of dissipation, and to follow the trajectories of a Lagrangian particle in order to find out the amount of dissipation and force a particle faces in the reactor. More recently, Brauckmann \& Eckhardt [22] presented detailed torque estimations and how they are influenced by the vortex heights in TVR for a radius ratio, ๆrr, of 0.71 using DNS model at various aspect ratios and shear Reynolds number up to $3 \times 104$.

Considering these various studies and the little information there is on the accurate estimation of VEDR in the TVR, the objective of this article is to further the numerical research by using the DNS to estimate the VEDR and its 12 terms in a TVR. An accurate estimation of the VEDR could be the essential parameter to design a bioreactor for culturing animal cells.

\section{Materials and Methods}

\subsection{Description of Case Set-up}

A TVR with inner, $r_{i}$, and outer, $r_{0}$, cylinder radius of 100 and $115 \mathrm{~mm}$, respectively, and a height, h, of $200 \mathrm{~mm}$ was used in this study. This leads to a gap width, $b=r_{0}-r_{i}$, radius ratio, $\eta_{\mathrm{rr}}=\mathrm{r}_{\mathrm{i}} / \mathrm{r}_{\mathrm{o}}$, and aspect ratio, $\Gamma=\mathrm{h} / \mathrm{b}$, of $15 \mathrm{~mm}$, 0.87 and 13.3, respectively. The angular velocity, $\omega$, of $11.94 \mathrm{rad} / \mathrm{s}$ is used in this study for both the DNS and PIV methods with the Reynolds number, $\mathrm{Re}=\mathrm{r}_{\mathrm{i}} \omega\left(\mathrm{r}_{\mathrm{o}}-\mathrm{r}_{\mathrm{i}}\right) / v$, and Taylor number, $\mathrm{Ta}=\mathrm{r}_{\mathrm{i}}^{1 / 2} \omega\left(\mathrm{r}_{\mathrm{o}}-\mathrm{r}_{\mathrm{i}}\right)^{3 / 2} / \mathrm{v}$, of 17900 and 6935 , respectively. Inner cylinder was placed at $0 \mathrm{~mm}$ above the outer cylinder. Water at at $20^{\circ} \mathrm{C}$ was used as the working fluid.

\subsection{Experimental Method}

The PIV system consists of a class IV Quantel Big Sky Laser $(15 \mathrm{~Hz}$ and $\lambda=532 \mathrm{~nm})$, FlowSense EO $16 \mathrm{M}$ camera $(4872 \times 3248)$ provided by Dantec Dynamics using a $60 \mathrm{~mm}$ objective having a diaphragm aperture of $\mathrm{f} / 2.8$ to $\mathrm{f} / 32$ and a synchronization system. The black colored internal cylinder is made of PVC, and the transparent external cylinder is made of Plexiglas. The camera was placed on the top of the TVR to the capture the motion of particles in the $\mathrm{X}-\mathrm{Y}$ plane. The $\mathrm{XY}$ plane is chosen in order to capture the radial flow structures and radial gradients, which are assumed to be the principal components of the EDR in a TVR. Capturing these smallest possible scales with regard to the radial gradients for a better estimation of EDR is the main reason for the usage of the 2D PIV measurements in the X-Y plane, instead of the 3D stereographic or tomographic PIV study which would have provided the out-of-plane motion but at the expense of the spatial resolution.

The first chosen location was dependent on the height at which the laser equipment can be located to illuminate the chosen $\mathrm{X}-\mathrm{Y}$ plane. Once the reactor and the laser equipment were put into place in order to capture the closest feasible X-Y plane, the camera and lens were focused in that respective plane. Between the camera and the chosen X-Y plane lays the fluid above that plane, and the flat and transparent Plexiglas plate placed on the top of the reactor. As the Plexiglas plate is flat and the motion imparted by the inner rotating cylinder is purely tangential which spread radially towards the outer cylinder, the perspective error in order to capture the tangential and the radial velocity components is assumed to be negligible in this case. Each further chosen location was $10 \mathrm{~mm}$ downwards which lead to a small decrease in the spatial resolution for each further location. The five chosen horizontal locations are between $\mathrm{Zh}=0.725 \pm 0.005$ and $0.525 \pm 0.005$.

Silver coated glass beads of $10 \mu \mathrm{m}$ size were used as seeding particles. The acquired data was processed with an image acquisition system provided by Dantec Dynamics, Dynamicstudio V3.31 The vector analysis was conducted using the adaptive correlation with a $50 \%$ overlapping window and interrogation cell size (ICS) of 16 pixels squared for high resolution, thus giving a spatial resolution of $68 \mu \mathrm{m}$. The spurious vectors were removed using range validation, and then these were replaced by moving average validation using 3 or 5 neighboring cells, in most cases 3 neighboring cells were used. A total of 2500 image pairs were found to be more than sufficient for each $\omega$ at five different horizontal locations to achieve the statistical convergence of the second order fluctuating velocity components, namely ur' $2, \mathrm{u} \theta^{\prime} 2$ and ur'u $\theta^{\prime}$. In this study, data for only one horizontal location is presented for the purpose of validation. This chosen location is 
$\mathrm{Z}_{\mathrm{h}}=\mathrm{z} / \mathrm{h}=0.725 \pm 0.005$ because it is the closest feasible location in the current set-up as explained above, thus the lowest spatial resolution available is chosen for better comparison between numerical and experimental results.

The acquired data was processed with an image acquisition system provided by Dantec Dynamics, Dynamicstudio V3.31. The vector analysis was conducted using the adaptive correlation with a $0 \%$ overlapping window and interrogation cell size (ICS) of 16 pixels squared for high resolution, thus giving a spatial resolution of $136 \mu \mathrm{m}$. The spurious vectors were removed using range validation, and then these were replaced by moving average validation using 3 or 5 neighboring cells, in most cases 3 neighboring cells were used. In total, less than $2.9 \%$ percent of spurious vectors were replaced at the $Z_{h}=0.725 \pm 0.005$.

\subsection{Numerical Method}

All of these simulations were performed on a desktop with six-core and twelve-thread $3.5 \mathrm{GHz}$ i7-3970X processor, and $64 \mathrm{~GB}$ of memory. All of the simulations were run using hpi based parallel processing with 4 cores. ANSYS-Fluent version 13.0.0 was used to perform the numerical simulations in the TVR.

\subsubsection{Geometry and Meshing aspects}

Workbench was used to construct geometry and the sweep mesh consisting of only hexahedral nodes. In view of the intended use of the DNS model, and multiple reference frame (MRF) technique in the modeling aspects, a full geometry was modeled containing only one body. However, in order to obtain better control over the radial mesh, the TVR geometry was designed as a semi-circle and was later on duplicated to model the full geometry. MRF technique was used instead of the more sophisticated but time-consuming sliding grid technique because the fluid movement in the TVR is only due to the inner-rotating cylinder; thus, avoiding the usage of an inner and outer geometrical configuration required for the sliding grid interface.

The mesh was constructed with high refinement in the radial direction with more nodes towards the boundaries then in the bulk zone to capture the large gradients towards the boundary layer area. The mesh was more refined in the radial direction because the secondary vortexes consisting of the radial and axial components of the velocity are only $10 \%$ of the tangential component [24]. This led to a mesh with 201 (axial covering $200 \mathrm{~mm}$ height $\times 151$ (radial covering $15 \mathrm{~mm}$ gap-width $) \times 320$ (tangential covering $360^{\circ}$ rotation $)=9.712$ million nodes with axial, radial min and max, and tangential spacing of $1 \mathrm{~mm}, 47$ and $184 \mu \mathrm{m}$, and $1.96 \mathrm{~cm}$, respectively. In terms of the global estimate of Taylor's micro-scale, $\Lambda=1.3$ $\mathrm{mm}$, the mesh is sufficiently fine both near the walls and in the bulk zone in the radial and axial directions to capture the large-eddies defined by the Taylor's microscale. However, the global estimate of Kolmogorov scale of $52.5 \mu \mathrm{m}$ shows the probability that near the wall and in the bulk zone it must be smaller than the $47 \mu \mathrm{m}$ and 184 $\mu \mathrm{m}$, respectively. This indicates that there could be some under-estimation of the VEDR especially towards the bulk zone where the grid size is comparatively bigger.

\subsubsection{Governing equations}

Please note that the equations used in the study are those that are described in detail in the Ansys-Fluent version 13.0.0. The DNS model has been used in this study in order to solve all the spatial and temporal scales of the turbulence of the Navier-Stokes equations of continuity and momentum for an incompressible flow, Newtonian fluid and zero external forces.

\subsubsection{Pre and post processing aspects}

The Convergence criterion of $10^{-4}$, which was achieved within a maximum of 40 iterations per time-step, for root mean square scaled residuals was employed for the continuity, momentum and turbulence quantities. Double precision arithmetic was applied for all of the simulations. In order to collect the data, an xz-plane was created, and a certain number of variables, including velocity components and gradients, were chosen to be extracted at the end of each time-step in the text file format. The high resolution and second order backward Euler schemes were used to model the advection and transient terms, respectively, for all the equations.

It took around 15 revolutions for the DNS model to achieve the moment stabilization, and 36 hours to complete one revolution. Once the moment stabilization was achieved, the data was collected for at-least 20 revolutions. The collected data was then treated using the Matlab program to process the results. As in the case of the experimental results, the statistical convergence of the second order fluctuating velocity components, namely $\mathrm{u}_{\mathrm{r}}{ }^{2}, \mathrm{u}_{\theta}{ }^{2}$ and $\mathrm{u}_{\mathrm{r}} \mathrm{u}_{\theta}{ }^{\prime}$ was also tested, and 20 revolutions or 7200 time-steps were found to be more than sufficient for an ensemble average.

\subsection{Estimation of VEDR}

The PIV measurements were taken in a horizontal plan in a location where $\mathrm{U}$ or $\mathrm{u}^{\prime}=\mathrm{U}_{\theta}$ or $\mathrm{u}_{\theta}^{\prime}$ represents mean and fluctuating tangential component and $\mathrm{V}$ or $\mathrm{v}^{\prime}=\mathrm{U}_{\mathrm{r}}$ or $\mathrm{u}_{\mathrm{r}}^{\prime}$ represents radial component of the velocity, and $\mathrm{x}$ and $\mathrm{y}$ implies tangential and radial directions, respectively, and were extracted as *.txt files from the image files. In the case of the numerical results, the axial, radial and tangential velocity and direction components were extracted along with the gradients of the velocity in the Cartesian co-ordinate system at each time step in the *.txt format. The txt files for the experimental results were treated with Matlab to create the gradients as per Cartesian co-ordinates using the $2^{\text {nd }}$ order central differencing approximation of the first derivative, Equation 1, for the central elements. The boundary 
elements being only at the inner and outer wall due to the usage of the $2^{\text {nd }}$ order scheme were not considered for the EDR estimation.

$f_{i}^{\prime}=\frac{f_{i+1}-f_{i-1}}{2 h}+E, E \quad \frac{1}{6}{ }^{2}\left(f_{i}^{3}\right)$

The gradients were estimated using the mean and fluctuating velocity components. The VEDR derived from the gradients of the mean and turbulence flow are hereonwards termed as the VEDR of MFKE and TKE, respectively. These two components of VEDR are estimated directly from the Equations 2 and 3, respectively, in the case of DNS model. In the case of PIV measurements only five out of 12 gradients are available from the $x-y$ plane.

$\epsilon_{m}=v\left\{2\left((\partial \bar{U} / \partial x)^{2}+(\partial \bar{V} / \partial y)^{2}+(\partial \bar{W} / \partial z)^{2}\right)+\right.$ $(\partial \bar{U} / \partial y)^{2}+(\partial \bar{U} / \partial z)^{2}+(\partial \bar{V} / \partial x)^{2}+(\partial \bar{V} / \partial z)^{2}+$ $(\partial \bar{W} / \partial x)^{2}+(\partial \bar{W} / \partial y)^{2}+2(\partial \bar{U} / \partial y . \partial \bar{V} / \partial x+$ $\partial \bar{U} / \partial z . \partial \bar{W} / \partial x+\partial \bar{V} / \partial z . \partial \bar{W} / \partial y)\}$

$\epsilon_{t}=v\left\{2\left(\overline{\left(\partial u^{\prime} / \partial x\right)^{2}}+\overline{\left(\partial v^{\prime} / \partial y\right)^{2}}+\overline{\left(\partial w^{\prime} / \partial z\right)^{2}}\right)+\right.$ $\overline{\left(\partial u^{\prime} / \partial y\right)^{2}}+\overline{\left(\partial u^{\prime} / \partial z\right)^{2}}+\overline{\left(\partial v^{\prime} / \partial x\right)^{2}}+\overline{\left(\partial v^{\prime} / \partial z\right)^{2}}+$ $\overline{\left(\partial w^{\prime} / \partial x\right)^{2}}+\overline{\left(\partial w^{\prime} / \partial y\right)^{2}}+2\left(\overline{d u^{\prime} / d y \cdot \partial v^{\prime} / \partial x}+\right.$ $\left.\left.\overline{\partial u^{\prime} / \partial z . \partial w^{\prime} / \partial x}+\overline{\partial v^{\prime} / \partial z \cdot \partial w^{\prime} / \partial y}\right)\right\}$

\section{Results and Discussion}

\subsection{Validation of the Computational Model}

The validation of the numerical results was done at only one of the five available horizontal locations $\left(Z_{h}=0.725 \pm\right.$ 0.005 ) of the PIV measurements. The comparison with the PIV data was conducted for the tangential and radial velocity components, squared fluctuating tangential velocity and the five available gradients of the VEDR of the MFKE and TKE for the PIV measurements, as shown in Figures 1, 2, 3, 4 and 5.

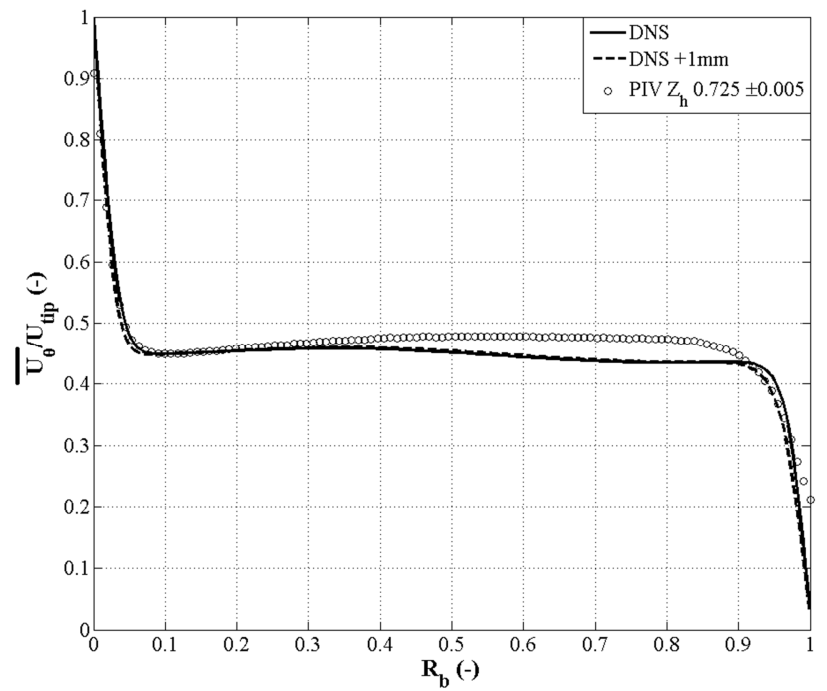

Figure 1: Comparison of the DNS model with the PIV results for the tangential velocity component.

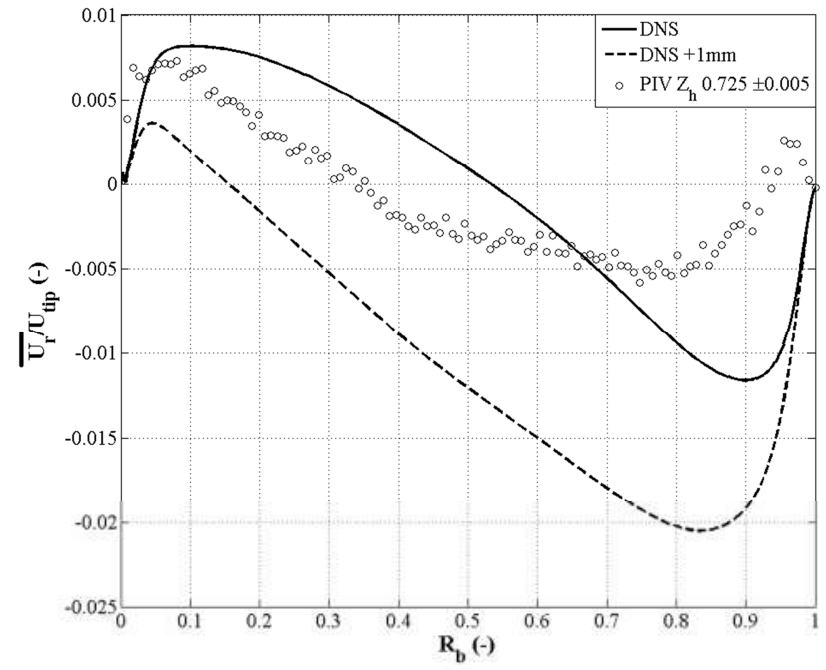

Figure 2: Comparison of the DNS model with the PIV results for the radial velocity component.

First of all, because the PIV location is not $100 \%$ certain, the numerical horizontal location was chosen by observing a complete Taylor-Vortex pair and thereby choosing the most similar profile to that of the PIV measurement. The PIV profile lies in between the center of vortex and outward flow region of a Taylor-Vortex, but in close proximity to the center of vortex. The term center of vortex means as it says and the outward flow region means the region when the flow direction is at its strongest from the inner cylinder towards the outer cylinder, which is the center of a Taylor-vortex pair not to be confused with center of vortex. Each Taylor-vortex pair has one Outward flow, which lies in the center of this Taylor-vortex pair, two center of vortexes, in each vortex of the pair, and two inward flow regions, at each extremity of the pair. Secondly, considering that the numerical mesh spacing is of $1 \mathrm{~mm}$ in the axial direction, Figures 1 and 2 present data at two axial locations for the numerical DNS model. Finally, in the Figures 4 and 5 $\varepsilon_{\text {innerwall }}=\mathrm{u}_{\mathrm{\tau i}}^{4} / \mathrm{v}$, where $\mathrm{u}_{\mathrm{\tau i}}=\left(\tau /\left(\rho 2 \pi \mathrm{r}_{\mathrm{i}}^{2} \mathrm{~h}\right)\right)^{1 / 2}$ where $\tau$ is torque and estimated using the DNS data.

The tangential velocity component (see Figure 1) is well predicted by the DNS model especially in the boundary layer; in addition, the difference between the axial heights is negligible which implies that this coarseness of mesh spacing in the axial direction has little impact on the tangential velocity component. In the bulk zone, between $\mathrm{Rb}=0.1$ to 0.9 , in agreement with Bilson \& Bremhorst [18], the numerical model does not accompany the phenomenon of small and steady increase through-out the bulk zone, as in the case of experimental results $[24,25]$ and Figure 1 , but only till $R_{b}=r$ (radial location $) / b$ (gap width $)=0.3$. Kobayashi et al. [24] stated that this phenomenon is because of uniformity in the circumferential velocity, in which the velocity profiles apart from the ones in the outward and inward region are very similar to the one in the center of vortex region, which in turn is caused by convection of the secondary 
flow. In-fact, none of the numerical studies [18, 20, 2628] demonstrated the capability of the various numerical models to capture this phenomenon exhibited by experimental methods. In the case of the radial component (see Figure 2), though the magnitude is less than $3 \%$ of the tangential component and the shape is well predicted by the numerical model, the maximum difference between the two axial heights is approximately $25 \%$. In order to better capture the radial component reduction in the axial mesh size to 0.5 or perhaps to $0.25 \mathrm{~mm}$ is required, implying doubling or quadrupling, respectively, the total number of nodes, which is impractical presently to implement with the available computational resources and, in turn, time.

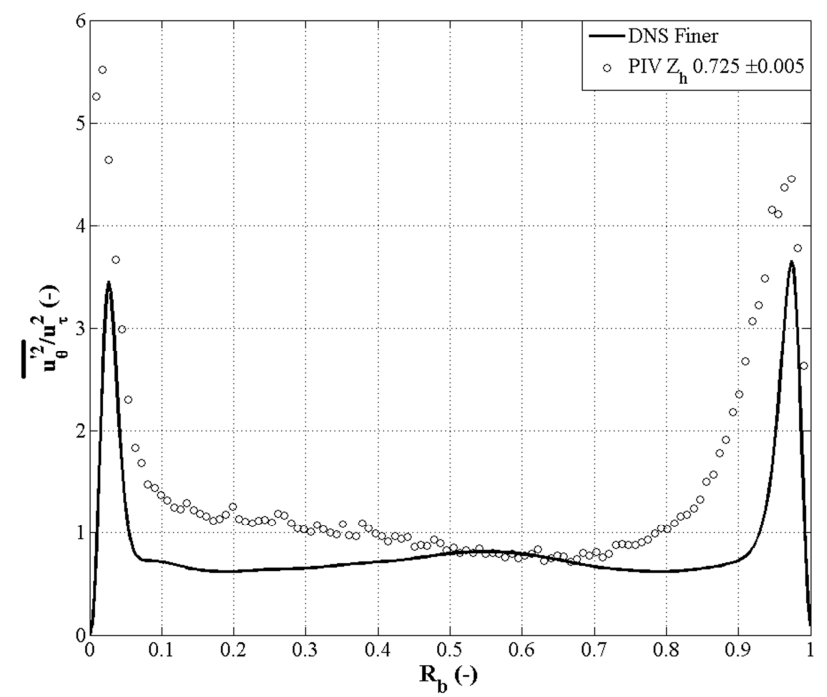

Figure 3: Comparison of the DNS model with the PIV results for the tangential normal stress component.

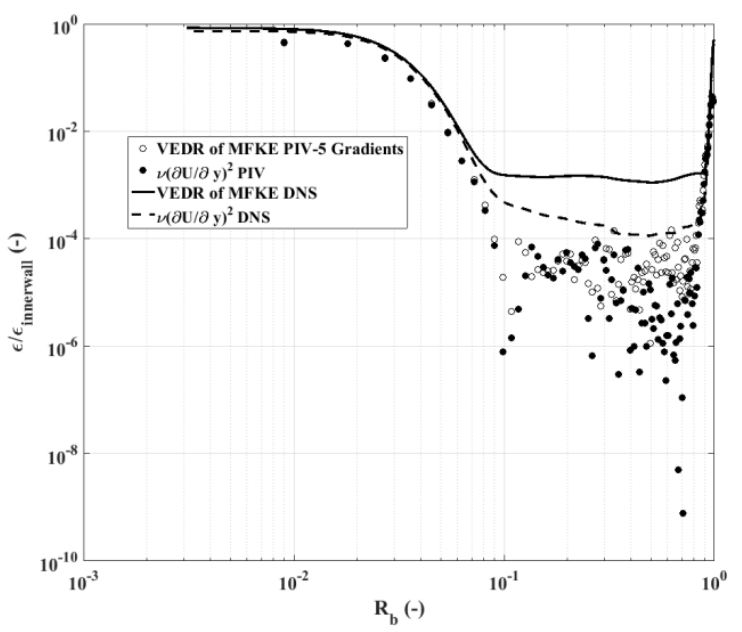

Figure 4: Comparison of the DNS model with the PIV results for the VEDR of the MFKE.

Proceeding towards the tangential normal stress component (see Figure 3), it can be observed that qualitatively the numerical model estimate it well. In the boundary layer of the inner cylinder, the DNS model estimates the maximum by around $60 \%$ of the PIV; while, towards the boundary layer of the outer cylinder the estimation improves significantly in comparison to the inner wall. In the bulk zone, the estimation by the numerical model is much better compared to the boundary layer, but still comparatively under-predicting. Chung \& Sung [26], Poncet, Haddadi and Viazzo [27] and Poncet, Viazzo \& Oguic [28] also observed similar results in the validation of their numerical models in both the boundary layer and bulk zone.

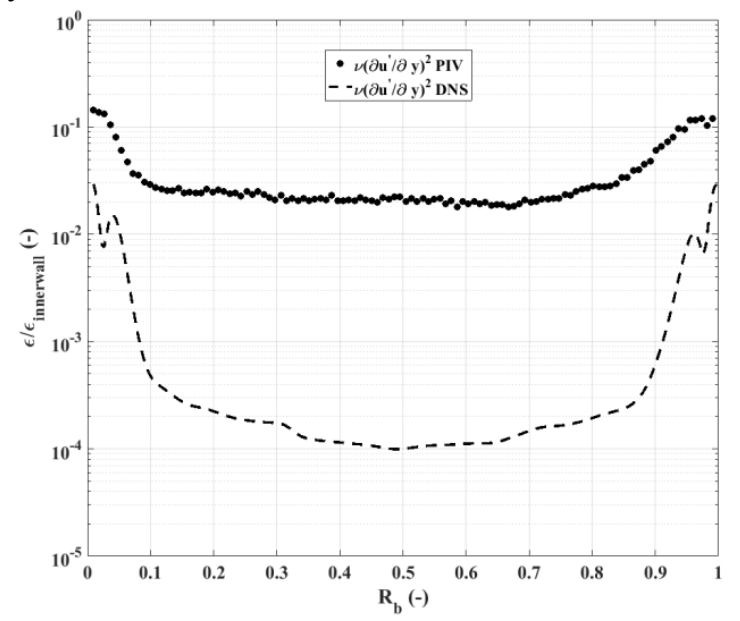

Figure 5: Comparison of the DNS model with the PIV results for the VEDR of TKE.

For the VEDR of the mean flow kinetic energy (see Figure 4), it can be seen that the numerical model predicts the magnitude and the structure well in comparison with the experimental PIV estimations, especially in the boundary layer where the estimation is even comparatively higher. The VEDR decreases rapidly and remains practically in the vicinity of 0.001 throughout the bulk zone for the DNS model and $10^{-5}$ for the PIV method. It should be remembered that the PIV estimations are based on only five gradients in comparison to the 12 gradients for the DNS model; additionally, the spatial resolution of $136 \mu \mathrm{m}$ remains constant through-out the grid structure for the PIV in comparison to the DNS model where the grid structure varies with least value being towards the walls $(47 \mu \mathrm{m})$ and the highest being towards the center of the gap-width $(184 \mu \mathrm{m})$. The $(\partial \mathrm{U} / \partial \mathrm{y})^{2}$ gradient can be seen as the principal component of the five-gradient viscous VEDR through-out the reactor for both the PIV estimations (hollow circles completely hidden behind dark circles in the inner boundary layer) and DNS simulations.

The $\left(\partial u^{\prime} / \partial y\right)^{2}$ gradient VEDR of turbulent kinetic energy (see Figure 5) suffers significantly from the comparatively coarser grid structure in the bulk zone for the DNS model. The maximum near the wall is also under-predicted but the discrepancy increases significantly towards the bulk zone, where the difference is more than 20 times. Although, the difference between the mesh structures of the DNS and PIV is just around 30 $\%$, this significant under-estimation in the bulk zone of 
the VEDR of turbulent kinetic energy in comparison to the VEDR of mean flow kinetic energy is due to the fact that the magnitude of the VEDR of turbulent kinetic energy is practically an order higher in the bulk zone in comparison to the VEDR of mean flow kinetic energy. In addition, the increase in the VEDR nearer to the Kolmogorov scale is much more drastic in comparison to away from the Kolmogorov scale as it follows a power law of the type: $\varepsilon /$ (normalisingfactor) = A(spatialresolution/GlobalKolmogorovscale) B; where A and $\mathrm{B}$ are coefficients of the power law over the chosen area, as shown by Delafosse et al. [17]. They found that if the spatial resolution decreases by $50 \%$, the corresponding increase in the VEDR of turbulent kinetic energy is over $220 \%$ for spatial resolution values approaching the global Kolmogorov scale.

In similar sense, the estimation of the VEDR of turbulent kinetic energy for the PIV measurements must be under-predicting as well, especially in the boundary layer area, considering the fact that the spatial resolution is of $136 \mu \mathrm{m}$ in comparison to the global Kolmogorov scale of $52.5 \mu \mathrm{m}$. The global Kolmogorov scale value indicates that its value must be smaller in the boundary layer area and comparatively higher in the boundary layer area. This was the idea that was kept in the back of the mind when the varied radial mesh was created for the DNS model. This mesh structure definitely requires further refinements not only in the radial direction but also in the axial and tangential directions, which would mean something above 100 million nodes for this current configuration, impractical in the present case scenario due to limited computational resources.

In the case of the PIV, using a higher overlap ratio would have given a smaller spatial resolution, but this smaller spatial resolution is just the manipulation of the base structure in order to increase the number of data points, therefore does not represent the true picture of a smaller spatial resolution. Moreover, smaller spatial resolution of the order of $50 \mu \mathrm{m}$ would require particles of much smaller size of 1-2 $\mu \mathrm{m}$, which are extremely costly, to achieve good concentration of particles in the fluid for the corresponding grid structure. To avoid these details, the base overlap ratio of $0 \%$ was employed in this study. Using a 32 Mpixels camera would have reduced the resolution by half but would also require smaller sized particles. This study presents the smallest spatial resolution for both the DNS and PIV study for the Reynolds number of 17900 . Additionally, as per author's knowledge, validation of the numerical model based on this direct VEDR estimation has not been conducted before-hand for the TVB.

\subsection{Composition of VEDR}

In Figure 6 the composition of the twelve gradients is shown for the estimation of VEDR of the MFKE and TKE for the DNS model. It can be seen that the major component near and away from the walls for both components of the VEDR is the gradient of the tangential velocity component in the radial direction: $(\partial \mathrm{U} / \partial \mathrm{y})^{2}$ for the VEDR of the MFKE and $\left(\partial u^{\prime} / \partial y\right)^{2}$ for the VEDR of the TKE. In combination with the gradient of axial velocity in the radial direction, i.e. $(\partial \mathrm{W} / \partial \mathrm{y})^{2}$, these account for at-least $90 \%$ of the VEDR of MFKE throughout the reactor. These two gradients of the radial direction practically constitute the VEDR of TKE also with the rest of the gradients being closer to lower than $10^{-4}$ of the $\varepsilon_{\text {innerwall }}$. The three gradients of the axial and tangential direction account for the less than $10^{4}$ and $10^{3}$ of the $\varepsilon_{\text {innerwall }}$ for the TKE and MFKE, respectively, through-out the reactor. Among the three cross product gradients, the gradient of the radial and axial velocity components is the strongest of the three and is of significant importance in the bulk zone for both components of the VEDR.
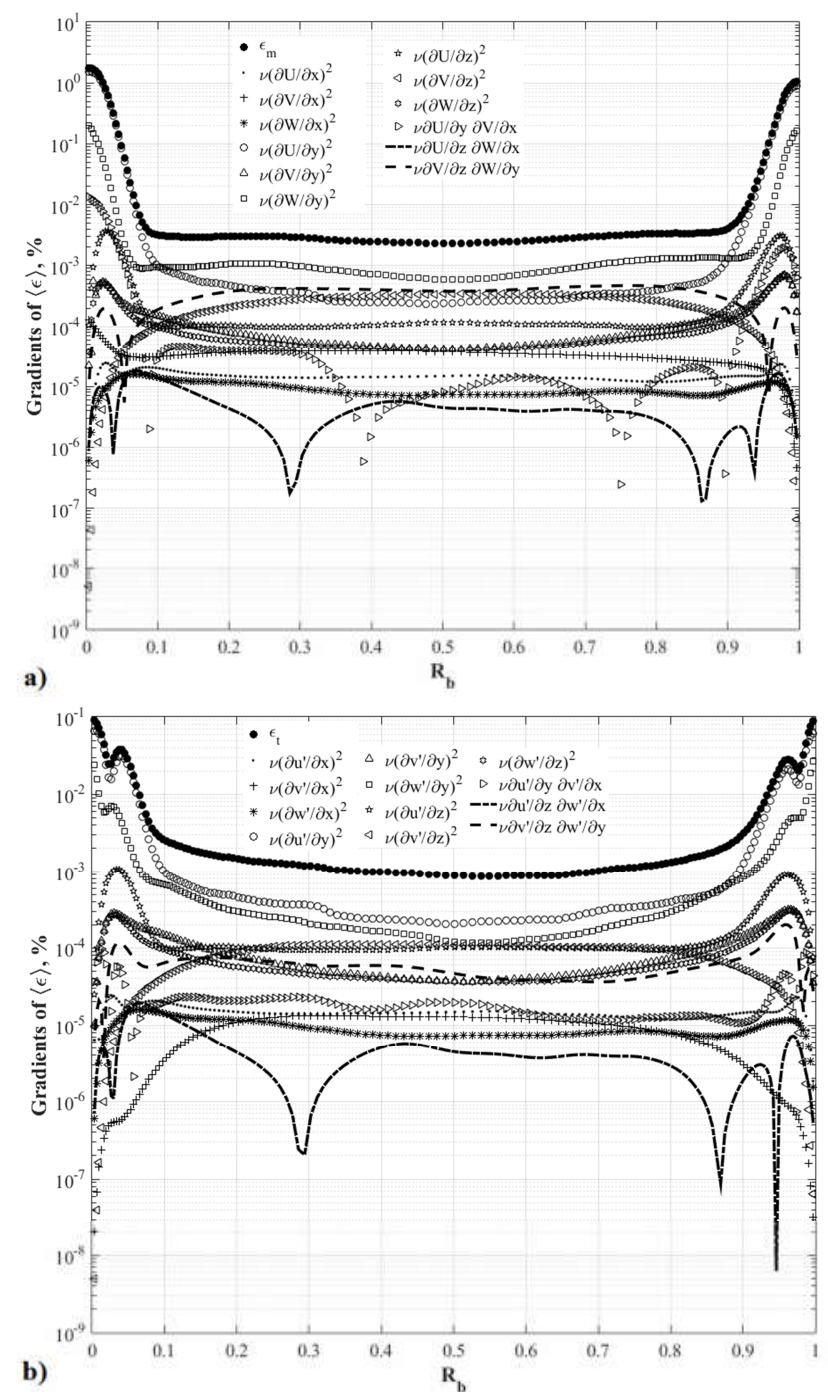

Figure 6: The twelve gradients of the VEDR of MFKE (a) and TKE (b) for the DNS model.

Sharp \& Adrian [29] presented equations based on the statistical isotropy or axi-symmetry to capture the outof-plane gradients for the 2D-PIV measurements. In these equations, an arithmetic mean of the in-plane gradients is 
taken into consideration to account for the out-of-plane gradients. Keeping in view the impact of radial gradients on the VEDR, such methodology will generate overestimation of the VEDR in the case of a TVR. Therefore, in-order to achieve a good estimation of at-least the maximum and average VEDR, the gradients of the radial co-ordinate have to be well captured. The well captured radial gradients can provide a good estimation of the VEDR, especially near the wall where lies the maximum VEDR and which will lead to a better averaged VEDR as well. However, from the numerical $3 \mathrm{~d}$ point of view, care still has to be taken when constructing the grid in the axial and tangential direction in order to keep a good aspect ratio and orthogonal quality.

However, it should be remembered that the grid structure in the tangential and axial directions are more than 10 and 5 times coarser near the boundary layer and bulk zone, respectively, in comparison with the radial direction. In comparison with the five gradient PIV estimation of the VEDR ( see Figure 3), it was observed that even the finer grid structure in the radial direction presented significant amount of under-estimation for the VEDR of TKE in the bulk zone. It could mean that such poor magnitude values of the gradients in the axial and tangential direction could also be due to the coarser grid structure in these directions. An improved mesh structure not only in the radial direction but also in the axial and tangential direction can shed further light on this subject.

\section{Conclusion}

The main aim of this article was to validate the DNS model estimations with experimental PIV results within a certain degree of acceptance and to further the research on the VEDR and its 12 terms. The grid structure was found to require improvements: firstly, in the axial grid as difference of $25 \%$ in the magnitude between two values of the radial velocity components at two consecutive axial heights $1 \mathrm{~mm}$ apart was observed; secondly, in the bulk zone because mesh size was found to be slightly on the courser side in the bulk zone in comparison to the Kolmogorov's microscale, and finally in the tangential direction as well due to grid spacing of only $1.96 \mathrm{~cm}$. These enhancements may not be feasible on present day desktop computers, however considering the speed of advancements in the computational resources, a completely grid-independent mesh should be available with reduced time within a decade thereby reducing the limitations due to mesh attained here.

Although, the ideal scenario of $100 \%$ concordance between the numerical and experimental study is not seen, the qualitative comparison between the two is completely acceptable due to good prediction of the structure and shape of the flow within the TVR. Quantitatively, the mean velocity predictions of the DNS are in good agreement with the PIV data; whereas, the fluctuating components of the velocity and the VEDR of turbulence kinetic energy are underestimated with major under predictions being in the boundary layer area for the fluctuating components and in the bulk zone for the VEDR of turbulence kinetic energy.
An important aspect of this study is the composition of the 12 gradients for the estimation of the VEDR. These compositions clearly showed that the two second-order radial gradients alone accounted for at-least $90 \%$ of the VEDR of mean flow and turbulence kinetic energy through-out the reactor; while, the cross-product gradients and the gradients of the tangential direction were found to have negligible impact. A CFD study gives access to the parameters which are not accessible with the 2D-PIV results, such as all 12 terms of the VEDR. Moreover, these numerical results are available through-out the geometry in complete contrast with the experimental results which are available only at a particular location for each experiment. The downside is the time of around 3-4 months to achieve statistically converged solutions for the DNS model, based on present computational resources.

\section{Acknowledgement(s)}

This study has become possible due to the postgraduate (processo no 140756/2012-4) and "Doutorado Sanduíche" (processo numero - 241739/2012-8) Scholarship programs of Conselho Nacional de Desenvolvimento Cientifico e Tecnologico (CNPq), Brasil.

\section{References}

[1] A.W. Nienow, Reactor engineering in large scale animal cell culture, Cytotechnology, 50, 2006, 9-33.

[2] S.J. Curran, \& R.A. Black, Oxygen transport and cell viability in an annular flow bioreactor: Comparison of laminar couette and taylor-vortex flow regimes, Biotechnology and Bioengineering, 89, 2005, 766-774.

[3] P.A. Santiago, R.d.C. Giordano, \& C.A.T. Suazo, Performance of a vortex flow bioreactor for cultivation of chok1 cells on microcarriers, Process Biochemistry, 46, 2011, 3545.

[4] N. Gregoriades, J. Clay, N. Ma, K. Koelling, \& J.J. Chalmers, Cell damage of microcarrier cultures as a function of local energy dissipation created by a rapid extensional flow, Biotechnology and Bioengineering, 69, 2000, 171-182.

[5] N. Ma, K. Koelling, \& J.J. Chalmers, Fabrication and use of a transient contractional flow device to quantify the sensitivity of mammalian and insect cells to hydrodynamic forces, Biotechnology and Bioengineering, 80, 2002, 428-437.

[6] M. Mollet, R. Godoy-Silva, C. Berdugo, \& J.J. Chalmers, Acute hydrodynamic forces and apoptosis: A complex question, Biotechnology and Bioengineering, 98, 2007, 772-788.

[7] M. Mollet, R. Godoy-Silva, C. Berdugo, \& J.J. Chalmers, Computer simulations of the energy dissipation rate in a fluorescence-activated cell sorter: Implications to cells, Biotechnology and Bioengineering, 100, 2008, 260-272.

[8] M. Mollet, N. Ma, Y. Zhao, R. Brodkey, R. Taticek, \& J.J. Chalmers, Bioprocess equipment: Characterization of energy dissipation rate and its potential to damage cells, Biotechnology Progress, 20, 2004, 1437-1448.

[9] R Godoy-Silva, J.J. Chalmers, S.A. Casnocha, L.A. Bass, \& N. Ma, Physiological responses of cho cells to repetitive hydrodynamic stress, Biotechnology and Bioengineering, 103, 2009a, 1103-1117.

[10] R. Godoy-Silva, M. Mollet, \& J.J. Chalmers, Evaluation of the effect of chronic hydrodynamical stresses on cultures of 
suspended cho-6e6 cells, Biotechnology and Bioengineering, 102, 2009b, 1119-1130.

[11] P. Mavros, Flow visualization in stirred vessels: A review of experimental techniques, Chemical Engineering Research and Design, 79, 2001, 113-127.

[12] S.T. Wereley, \& R.M. Lueptow, Spatio-temporal character of non-wavy and wavy taylor-couette flow, Journal of Fluid Mechanics, 364, 1998, 59-80.

[13] C. Coufort, D. Bouyer, \& A. Liné, Flocculation related to local hydrodynamics in a taylor-couette reactor and in a jar, Chemical Engineering Science, 60, 2005, 2179-2192.

[14] R. Deng, D.Y. Arifin, M.K. Chyn, \& C.H. Wang, Taylor vortex flow in presence of internal baffles. Chemical Engineering Science, 65, 2010, 4598-4605.

[15] R. Van Hout, \& J. Katz, Measurements of mean flow and turbulence characteristics in high-reynolds number counterrotating taylor-couette flow, Physics of Fluids, 23, 2011, 105102-11.

[16] S. Tokgoz, G.E. Elsinga, R. Delfos, \& J. Westerweel, Spatial resolution and dissipation rate estimation in taylorcouette flow for tomographic piv, Experiments in Fluids, 53, 2012 561-583.

[17] A. Delafosse, M.L. Collignon, M. Crine, \& D. Toye, Estimation of the turbulent kinetic energy dissipation rate from 2d-piv measurements in a vessel stirred by an axial mixel ttp impeller, Chemical Engineering Science, 66, 2011, 1728-1737.

[18] M. Bilson, \& K. Bremhorst, Direct numerical simulation of turbulent taylor-couette flow, Journal of Fluid Mechanics, 579, 2007, 227-270.

[19] S. Dong, Direct numerical simulation of turbulent taylorcouette flow, Journal of Fluid Mechanics, 587, 2007, 373-393.

[20] D. Pirrò, \& M. Quadrio, Direct numerical simulation of turbulent taylor-couette flow, European Journal of Mechanics B/Fluids, 27, 2008, 552-566.
[21] M. Douaire, Etude experimentale et numerique de la response de Lactococcus Lactis NCDO2118 aux conditions hydrodynamiques locales en reacteur Couette, Ph.D. thesis, Institut National des Sciences Appliquées (INSA) de Toulouse, 2010.

[22] H.J. Brauckmann, \& B. Eckhardt, Direct numerical simulations of local and global torque in taylor-couette flow up to re $=30$ 000, Journal of Fluid Mechanics, 718, 2013, 398-427. [23] M. Quadrio, \& P. Lichini, Direct numerical simulation of the turbulent flow in a pipe with annular cross-section, European Journal of Mechanics B/Fluids, 21, 2002, 413-427.

[24] M Kobayashi, H. Maekawa, T. Takano, \& Y. Yamada, An experimental study on turbulent taylor vortex flow between concentric cylinders, JSME International Journal II, 33, 1990, 436-445.

[25] G.P. Smith, \& A.A Townsend, Turbulent couette flow between concentric cylinders at large taylor numbers, Journal of Fluid Mechanics, 123, 1982, 187-217.

[26] S.Y. Chung, \& H.J. Sung, Large-eddy simulation of turbulent flow in a concentric annulus with rotation of an inner cylinder, International Journal of Heat and Fluid Flow, 26, 2005, 191-203.

[27] S. Poncet, S. Haddadi, \& S. Viazzo, Numerical modeling of fluid flow and heat transfer in a narrow taylor-couettepoiseuille system, International Journal of Heat and Fluid Flow, 32, 2011, 128-144.

[28] S. Poncet, S. Viazzo, \& R. Oguic, Large eddy simulations of taylor-couette-poiseuille flows in a narrow-gap system, Physics of Fluid, 26, 2014, 105108-1:27.

[29] Sharp, K.V., Adrian, R.J., 2001. Piv study of small-scale flow structure around a Rushton turbine. American Institute of Chemical Engineers - AIChE 47, 766-778. 